\section{Catching and Correcting Unreported, Under- Reported Accidents (Near-Misses) among Healthcare Provider in Thailand}

\section{Abstract}

Objectives: Latent errors in an incident reporting system pose threats to accident and near-miss prevention in hospitals. The aims of this study were to gain insight into the incident reporting system by exploring and investigating the refinement of unreported and under-reported (near-miss) patterns and by estimating underreported annual hospital accidents over three months in one hospital.

Methods: Sequential mixed-method research was undertaken using both qualitative and quantitative approaches. 120 health care providers were selected from 13 departments of a selected study hospital. Self-reported questionnaires, information from annual reports and focus group interviews among stakeholders were employed. Based on a quantitative mixed-model approach, estimation of lost cases from near-miss incidents was made.

Results: In 2015, 20\% of accidents had been reported to the hospital center while under-reported accidents and near-miss incidents by self-report over 3 months equaled $18 \%$ and $25.9 \%$, respectively. Recent trends were positive, driven by changing values about incident reporting. However, confusion and fear still remain among practitioners about near-miss reporting due to old beliefs. This study confirms that incident reporting needs improvement so that there is an enhanced organizational culture of safety, raised awareness for individual reporting, and recovery of lost cases using mixed-model estimation of near-misses.

Keywords: Accidents at work; Near-misses; Underreported; Health care provider

Received: March 14, 2017; Accepted: March 20, 2017; Published: March 27, 2017

\section{Significance and background}

Over a decade ago, the Institute of Medicine brought national and international attention to the issue of preventing patient harm through improving patient safety in its report. Today, reduction of medical errors continues to be a national priority [1]. One of the formal strategies widely utilized to address this issue is the use of incident reporting systems. Recording and reporting accidents, near-misses and ill health at work is a legal requirement under The Reporting of Injuries, Diseases and Dangerous Occurrences Regulations (RIDDOR) 2013 [2], reporting regulations for injuries, diseases and dangerous occurrences through risk management centers in hospitals with results aggregated in reports at the national level. According to recent research, a significant challenge remains in identifying and analyzing errors of all accidents and near-misses which obscure

\section{Pimpan Silpasuwan ${ }^{1}$, Chukiat Viwatwongkasem ${ }^{2}$, Pornpimol Kongtip ${ }^{3}$, Adul Bandhukul ${ }^{4}$, Thida Omas ${ }^{5}$ and Susan Woskie ${ }^{6}$}

1 Department of Public Health Nursing, Faculty of Public Health, Mahidol University, Bangkok, Thailand

2 Department of Biostatistics, Faculty of Public Health, Mahidol University, Bangkok, Thailand

3 Department of Occupational Health and Safety, Faculty of Public Health, Mahidol University, Bangkok, Thailand

4 Occupational and Environmental Institute, Kunnayoua District, Bangkok, Thailand

5 Occupational and Environmental Institute, Kunnayoua District, Bangkok, Thailand

6 Department of Work Environment, University of Massachusetts Lowell, USA

Corresponding author: Pimpan Silpasuwan

”pimpan.sil@mahidol.ac.th

Department of Public Health Nursing, Faculty of Public Health, Mahidol University, Bangkok, Thailand.

Tel: 668-356-19554

Citation: Silpasuwan P, Viwatwongasame C, Kongtip P, et al. Research Title: Catching and Correcting Unreported, Under-Reported Accidents (Near-Misses) among Healthcare Provider in Thailand. Arch Med. 2017, 9:2.

performance for future reporting of under and unreported incidents. Different accident and near-miss reporting patterns in different hospitals result in variation in unreported and underreported incidents, so it is difficult to know the exact volume of adverse events missed, the level of harm and what actual consequences occur and need addressing. Significantly, reports 
provide some insight into the underlying problems and need for prevention by safety stakeholders, including recognition of the need to provide important feedback information for knowledge sharing and prevention. The dominant reason that events aren't reported is that they aren't perceived as adverse events. Hospital personnel can be particularly vulnerable to errors due to the nature of their work; work with multiple tasks, multiple team members, and multiple interruptions. The reporting of accidents and near-misses is potentially an important part of many organizations' safety management systems [3], offering opportunities to monitor performance and create positive safety attitudes and increased alertness. Generally, such reports are registered in databases and analyzed in order to reveal trends and to help prioritize resources and accident prevention efforts $[4,5]$. Catching and correcting unreported incidents of accidents, especially the actual number of near-misses as precisely as possible, is considered crucial to prevent accidents [5].

The World Health Organization (WHO) published guidelines regarding safe practices in hospitals in 2009 [6], including citing various attempts made to improve patient safety such as preventing incidents of mistakes on the wrong patient or at the wrong site [4]. Hospital staff had to report their work related accidents, ill health, incidents and near-misses to the local central accident reporting system of the hospital, not only for on-site safety and risk management but also for organizational performance improvement overall. Reporting work related accidents, ill health, incidents and near- misses to the local reporting system online and electronically to the health and safety committee or risk management center of the hospital is a useful part of the investigative process which combines the requirements of social security and health and safety regulations [7].

In a previous study of the performance of hospital environmental service workers, $29.2 \%$ of the workers recalled being injured in the previous year on the job, but 38.9 percent did not report the injury, 64.4 percent of the unreported injuries required medical care and 44.1 percent resulted in lost work time [8]. Follow-up investigations revealed that total injuries were underreported by 11 percent, while lost workdays were underreported by $22 \%-23 \%$ [9]. One might assume that the same problem of underreporting occurred in 1997, 1998, 1999, and 2000. When hospital accidents and near-misses remain unreported or underreported, patient safety is increasingly threatened. Error reporting is known to be the best and most fundamental means of preventing medical errors [10]. The philosophy of a safe and healthy workplace has shifted. The occurrence of accidents or near-misses clearly signal the need for organizational improvement of unreported and underreported accidents and spotlight a significant opportunity to strengthen an organization's systematic capabilities to prevent future errors $[11,12]$. Although potential future medical errors can best be prevented through reporting accident and nearmisses, recent evidence shows that on-site error reporting is not being achieved to a satisfactory level in hospital settings [13].

The aims of this study are to gain insight into incident reporting systems of health care providers by investigating the refinement of unreported and underreported (near-miss) patterns and to describe the roots and functions of accidental reported or underreported of health care providers in a hospital in Thailand as Figure 1.

\section{Material and Methods}

\section{Sample selection and material}

To meet the measurement objectives for a hospital staff profile survey, mixed research methods were employed for a quantitative and sequential integration by qualitative means. For the quantitative part, a proportion of total health care services offered to the hospital service users required selecting a valid sample of the total unit in each service site in a Bangkok-area hospital in 2015-2016. An initial sample of health care providers were screened from the name lists of 21 departments providing tertiary care from the study hospital data-base of services which were of particular interest and with providers who gave consent to participate in this study. Representative health care providers with both direct and indirect contact or service with patients/clients were the sample base for the inclusion criteria. The self-completed questionnaires were sent to the recruited participants in the first phase of this study. In the second phase, semi-structured interview questions addressed wider issues indepth in order to receive information in focus group interviews. Individual in-depth interviews of key respondents such as hospital director and health care service practitioners included questions such as: "Why were accidents unreported or underreported by health care workers even with a safety, health- at-work policy? Including differentiated of an accident un-report and underreport meaning. In particular, we probed values about and beliefs regarding an acknowledgement of errors with repercussions for the professional credibility of health care service quality and including any expression of anxiousness, or feelings of guilt or fear about willingness to report accidents (near-misses) ".

\section{Data collection}

Following Institutional Review Board (IRB) approval of the study protocol (2015) in accordant with the Helsinki Declaration as revised in 2013, the 120 selected hospital health care providers (with direct and/or indirect patient contact) working in 13 departments directly received questionnaires from the researcher

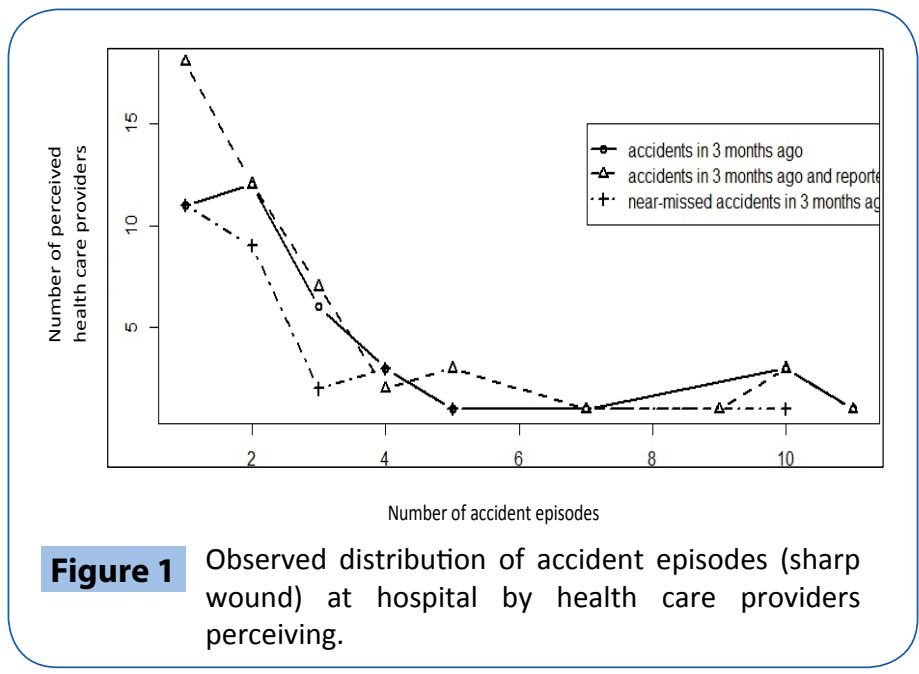


team regarding the frequency of accident and near-miss occurrence in the previous 3 months, and whether or not they reported the adverse events to the risk management center of the hospital. Assessment items included: occurrence of accidents or near-misses in the last 3 months, type of accident, willingness to report accidents and near-misses, and annual hospital reports from the risk management center. Apart from the questionnaire, a qualitative research approach was also used; Data was collected from key stakeholders by in-depth, focus group interviews and through discussion with direct and in direct health care providers and the hospital director who were a significant part of safety practices during the same six month period of time. All research instruments were assessed for validity and reliability by 3 experts before implementing the data gathering.

\section{Data analysis}

A modified, manual statistical equation to calculate an estimate of underreported hospital accidents in 3 months was used. Using a quantitative approach, a mixture of truncated Poisson models were employed for estimating the lost cases of accidents and near-misses reported [14]. The best component mixture with the smallest value of the Bayesian Information Criterion (BIC) was selected to be an appropriate model among various component mixtures. The Horvitz-Thompson approach [15] led to a population size and lost cases number estimation. Aside from this qualitative approach, an inductive content analysis on the data extraction was applied to the interview content record verbatim from stakeholders. Due to the exploratory content being extracted and its essence narrowed down to main- and sub-themes, an inductive, interpretive approach was employed for analysis of the essence of data.

\section{Results}

A total number of 120 of health care provider in the hospital participated in this study, most of them (78.5\%) were direct practice with patients, having work experiences in hospital more than 10 years as Table 1.

Only $20 \%$ of health care provider receiving the accidental problems related work. The observed variables of incidents were classified by the characteristics of the accident self-reports during the previous 3 months such as sharp wound (20.8\%), needle stick Table 2.

To estimate the total number of accidents and near-misses from lost cases, unreported and underreported, a mixture of truncated Poisson models was applied. The result show that the best fit, the smallest BIC value was a mixture with two components of zerotruncated Poisson distribution. The rationale of the calculation was to take the number of occurred accidents in a health care provider and multiply it by the number of accidental episode per case as Tables 3-5.

The results produced missing, underreported, incidents at 7 cases for the unobserved sizes at zero-accident time (with no written records to the risk management hospital center), while the reported accidents to the risk management center were 38
Table 1 The number and percentage of the health care providers; personal characteristics $(n=214)$.

\begin{tabular}{|l|c|c|}
\hline personal characteristics & Number (case) & Percentage \\
\hline $\begin{array}{l}\text { Health service } \\
\quad \begin{array}{l}\text { Direct practice with patient } \\
\text { In-direct practice with patient }\end{array}\end{array}$ & 168 & 78.5 \\
\hline $\begin{array}{l}\text { Gender } \\
\quad \text { Female }\end{array}$ & 46 & 21.5 \\
$\quad$ Male & 190 & 88.8 \\
Work experiences & 24 & 11.2 \\
$\quad$ Under 5 years & 46 & \\
$\quad$ 6-10 years & 35 & 21.5 \\
$\quad$ More than 10 years & 133 & 16.4 \\
\end{tabular}

cases. Therefore, the total possible cases of hospital accidents that occurred were 45 cases for the total number of accidents in 3 months in the year 2015.

(The technical calculation methods for Tables 3-5 is shown in appendix section 1). Under the Broos trapping method, the associated $95 \%$ confidence interval for the total hospital accident frequency during the previous 3 months was between 40.3 and 49.7 cases. Underreported accidents (near-misses) were $18 \%$ and $25.9 \%$, respectively over the 3 month period. For near-miss around $31.31 \%$ of them identify receiving near miss but no any reported because of did not clear understanding the definition of terms and not sure whether or not have to be reported.

About the qualitative data reflected that intention to report all kind of accident to the committee of the risk management center in the hospital, the hospital director stated, "They all accept that accidents and injuries still exist in the hospital, almost reaching $20 \%$ which is the same as in the last 3 years with no decline. This persistent level is because of the many accident and injury campaigns in previous years, including the hospital safety training program on accidents and near-miss prevention and reports, as well as the surveillance system interventions for incident prevention." Thus, the hospital director summarized his mood of confidence in the ongoing processes of accident reported was in positive trends among health care officer as his expressed that, "Many hospital officers know more about the value of accident reporting. "No Blame is a good new value for incident reporting leading to their change in thinking; that's why in these years (2015-2016), the accident rate is not decreasing". This is an indication that there is a new acceptance about accidents which has already shifted among leaders and practitioners. Apart from this, one view from a hospital department head reluctantly expressed stated, "Some of the hospital officers, especially in the practitioners' group, they really didn't know when they should or should not report accidents, and the near-misses also. This confusion shows the need to verify and clarify this situation, because the scope of the near-miss event concept and practice about accidents is not clear. Moreover, I sincerely see that some of them are still scared of any kind of reporting."

It's possible to estimate that accidents and near-miss unreported and underreported incidents still exist because officers are not clear whether and under what conditions they must report. 
Table 2 The number and percentage of incidents and accidents reported in the last three months of health care providers in each group ( $\mathrm{n}=214$ ).

\begin{tabular}{|c|c|c|c|c|c|c|c|c|}
\hline \multirow{2}{*}{$\begin{array}{l}\text { Accidents among health care } \\
\text { providers }\end{array}$} & \multirow{2}{*}{$\begin{array}{c}\text { None } \\
\text { Number } \\
\text { (person) }\end{array}$} & \multirow[b]{2}{*}{ percentage } & \multicolumn{2}{|c|}{ Having accident } & \multicolumn{2}{|c|}{ Unreported } & \multicolumn{2}{|c|}{ Reported } \\
\hline & & & $\begin{array}{l}\text { Number } \\
\text { (person) }\end{array}$ & percentage & $\begin{array}{l}\text { Number } \\
\text { (person) }\end{array}$ & percentage & $\begin{array}{l}\text { Number } \\
\text { (person) }\end{array}$ & percentage \\
\hline \multicolumn{9}{|l|}{ Direct practice with patient } \\
\hline Sharp / cut. & 133 & 79.2 & 35 & 20.8 & 31 & 88.6 & 4 & 11.4 \\
\hline Needle stick injuries & 152 & 90.5 & 16 & 9.5 & 14 & 87.5 & 2 & 12.5 \\
\hline Electric shock / shock & 166 & 98.8 & 2 & 1.2 & 2 & 100 & 0 & 0 \\
\hline Fall / crash landing & 164 & 97.6 & 4 & 2.4 & 3 & 75 & 1 & 25 \\
\hline Slide & 150 & 89.3 & 18 & 10.7 & 16 & 88.89 & 2 & 11.11 \\
\hline $\begin{array}{l}\text { Chemicals / fluids splashing into } \\
\text { eyes/body. }\end{array}$ & 138 & 82.1 & 30 & 17.9 & 26 & 86.67 & 4 & 13.33 \\
\hline Others & 160 & 95.2 & 8 & 4.8 & 6 & 75 & 2 & 25 \\
\hline \multicolumn{9}{|l|}{ In -Direct practice with patient } \\
\hline Sharp / cut. & 42 & 91.3 & 4 & 8.7 & 4 & 100 & 0 & 0 \\
\hline Needle stick injuries & 45 & 97.8 & 1 & 2.2 & 0 & 0 & 1 & 100 \\
\hline Electric shock / shock & 46 & 100 & 0 & 0 & - & - & - & - \\
\hline Fall / crash landing. & 46 & 100 & 0 & 0 & - & - & - & - \\
\hline Slide & 43 & 93.5 & 3 & 6.5 & 2 & 66.67 & 1 & 33.33 \\
\hline $\begin{array}{l}\text { Chemicals / fluids splashing into } \\
\text { eyes/body. }\end{array}$ & 45 & 97.8 & 1 & 2.2 & 1 & 100 & 0 & 0 \\
\hline Others & 46 & 100 & 0 & 0 & - & - & - & - \\
\hline
\end{tabular}

Table 3 Data for the number of accidental episodes(sharp wound) in $(i)$ and their number of cases $\left(n_{i}\right)$ in various situations.

\begin{tabular}{|c|c|c|c|c|c|c|c|c|c|c|c|}
\hline \multicolumn{12}{|c|}{ Having accidents (sharp wound)in previous 3 months } \\
\hline$i$ & 1 & 2 & 3 & 4 & 5 & 6 & 7 & 8 & 9 & 10 & 11 \\
\hline$n_{i}$ & 11 & 12 & 6 & 3 & 1 & - & 1 & - & - & 3 & 1 \\
\hline \multicolumn{12}{|c|}{ Having accidents (sharp wound)in previous 3 months and reported } \\
\hline$i$ & 1 & 2 & 3 & 4 & 5 & 6 & 7 & 8 & 9 & 10 & 11 \\
\hline$n_{i}$ & 18 & 12 & 7 & 2 & 3 & - & 1 & - & 1 & 3 & 1 \\
\hline \multicolumn{12}{|c|}{ Having near-missed accidents(sharp wound) in previous $\mathbf{3}$ months } \\
\hline$i$ & 1 & 2 & 3 & 4 & 5 & 6 & 7 & 8 & 9 & 10 & 11 \\
\hline$n_{i}$ & 11 & 9 & 2 & 3 & 1 & - & - & - & - & 1 & - \\
\hline
\end{tabular}

Table 4 The appropriate results of 2-components mixture models for estimating the lost cases and all accidental cases in various situations.

\begin{tabular}{|c|c|c|c|c|c|c|}
\hline & $\hat{\lambda}_{j}$ & $\hat{q}_{j}$ & log-likelihood & BIC & $\hat{n}_{0}$ & $\hat{N}$ \\
\hline \multicolumn{7}{|c|}{ Having accidents (sharp wound)in previous 3 months } \\
\hline 1 & 1.7228 & 0.8554 & -70.6309 & 152.1745 & 7 & 45 \\
\hline 2 & 9.0639 & 0.1446 & & & & \\
\hline \multicolumn{7}{|c|}{ Having accidents (sharp wound)in previous 3 months and reported } \\
\hline 1 & 1.507441 & 0.835922 & -88.2836 & 188.1808 & 11 & 59 \\
\hline 2 & 8.301397 & 0.164078 & & & & \\
\hline \multicolumn{7}{|c|}{ Having near-missed accidents(sharp wound) in previous 3 months } \\
\hline 1 & 1.5647 & 0.9551 & -41.6565 & 93.2004 & 7 & 34 \\
\hline 2 & 9.0215 & 0.0449 & & & & \\
\hline
\end{tabular}


Table 5 Estimating the lost cases and total number of accidental cases (sharp wound)classified by characteristics of accidental reports during previous 3 months.

\begin{tabular}{|c|c|c|c|c|c|}
\hline $\begin{array}{c}\text { Accidental characteristics(sharp } \\
\text { wound) }\end{array}$ & Observed cases $(n)$ & $\begin{array}{c}\text { Estimate of lost cases } \\
\qquad n_{0}\end{array}$ & $\begin{array}{l}\text { Estimate of total } \\
\text { cases }(\hat{N})\end{array}$ & $\begin{array}{l}95 \% \mathrm{Cl} \text { based on } \\
\text { normal approach }\end{array}$ & $\begin{array}{l}95 \% \mathrm{Cl} \text { based on } \\
\text { percentile approach }\end{array}$ \\
\hline $\begin{array}{l}\text { Having accidents in previous } 3 \\
\text { months }\end{array}$ & 38 & ( & 45 & $(39.6,50.5)$ & $(42.0,52.0)$ \\
\hline $\begin{array}{c}\text { Having accidents in previous } 3 \\
\text { months and reported }\end{array}$ & 48 & 11 & 59 & $(47.4,70.6)$ & $(54.0,76.0)$ \\
\hline $\begin{array}{l}\text { Having near-missed accidents in } \\
\text { previous } 3 \text { months }\end{array}$ & 27 & 7 & 34 & $(12.0,56.0)$ & $(31.0,72.1)$ \\
\hline
\end{tabular}

Moreover, there may be reluctance to accept the new value of accident and near-miss-reporting in the safety-organization culture in this transitional period since it contradicts the past safety policy and practicability of "safe practice, no more mistakes".

\section{Discussion}

The crucial types of accidents and near misses occurrence (in previous 3 months) and reported by hospital health care officers to the hospital risk management center in 2015 were shown that around $20 \%$ of the total from self-reports in the last 3 months. There were from needle sticks, sharp injuries and secretion contaminations, and others related to medications or working conditions. While as the accident reported or under reported or unreported were differentiated by the reasons of health care providers' perceptions that the most of accident under reports (16.8\%) are forgetting to report or thinking that it's trivia events or its preventable situation, therefore in this study the incident accident remains only underreported. In general they intend to report any kind of accident related work in every time due to the modernized concept of incident accident in hospital to be changes, the health care officers realized that accident reported is necessary condition for protecting staff and patient in negative consequence from accidents, except in case of minor problems and near miss event also because of their perception that its unnecessary to be reported and including reluctant to do so.

The model for estimation of near miss incidents, and underreported incidents, in hospital among 13 departments shows that in 3 months original self-reports, and underreported accidents were $18 \%$ and $25.9 \%$ respectively. This number means that the organizational climate and staffing produce similar needle stick injuries as among US nurses and adverse events in psychiatric care in US hospitals [16-18].

An analysis of stakeholder views about hospital accidents and near-miss reporting shows the view that the trend is for accidents to gradually decline as shown in the hospital annual report of 2015; the proportion of high severity accidents of accident occurrence has declined with fewer severe cases and milder cases rising to 83.17 compared to 80.2 in 2014 . It is likely that this is a good sign from the accident surveillance and management indicators of the hospital risk management center However, evidence still indicates the need to improve the safety conditions in the hospital since the incident reporting system requires that all health-care officers report any incident or accident including near-misses regardless of the harm incurred by the patient with officers using an electronic incident reporting system and also on paper immediately or in 24 hours after occurrence of the accident or near-miss to the risk management center $[19,20]$. The hospital staff is required to provide the following information: date, time, location, type of service, type of accident or near miss (for example: wrong time, wrong drug, wrong patient, any omissions) and severity of the error impacting the personal property environment or organization, and the number of people involved directly or indirectly in each incident. In addition, demographic characteristics of those reporting the incident and demographic and clinical characteristics of patients involved were also reported to the risk management center. The person who reported the incident did not receive any penalty following the newly-established core value of hospital safety practice focusing on "No blame to gain more benefit in preventing adverse effects" [20,21]. This is a new hospital policy instituted as a modern, future-oriented perspective for a leading tertiary care hospital. This is congruent with a 2005 US requirement which specifically addresses these concerns, the Patient Safety and Quality Improvement Act of 2005 [16]; the predictor variables emphasized were attitude toward reporting, social pressure to report, and perceived control over reporting.

This is congruent with what quality assurance research and regulations warn against $[17,18,22]$, that reporting not cause any immediate harm. The occurrence of near-misses clearly signals the need for organizational improvement and creates a significant opportunity for strengthening the organization's systematic capabilities, in terms of revealing the type of management and method of resource allocation that the organization must adopt in order to prevent future errors [23]. Near-miss or accident research is necessary because of the early detection of misconduct, safety and organizational deficiencies as well as for the identification of potential risks on an objective basis [16]. However, mandatory reporting is uncommon and, because of the large variety of reporting mechanisms and indicators [22], often leads to a lack of understanding regarding the current status of error reporting with few attempts made to conduct multidimensional analyses. Therefore, the occurrence of a minor error or near-miss is usually followed by connivance or silence rather than reporting [22]. The most accidents occurred among nurses, with needle sticks having the highest incident rate [22]. Nurses explained not reporting accidents as a defensive silence, a conscious individual mechanism to protect oneself from possible disadvantages under uncertain conditions and as a trigger of fears 
regarding the expected consequences of reporting. Therefore, in order to increase the willingness of hospital officers to report near misses, hospital managers must prioritize efforts to form an organizational culture that supports reporting. Near-miss and accident research involves early detection of misconduct, safety and organizational deficiencies as well as the identification of potential risks on an objective basis [23-25]. This study confirms that Thailand's RIDDOR regulations provide optimal balance of risk management priorities, but the record keeping system suffers from chronic unreported and underreporting of incidents potentially contributing to superior safety performance in Thailand RIDDOR's recordable classification [2] threshold which captures cases with 3 or more days away from work and with serious injuries (for example, amputations). However; the new trend of increasing incident reports to prevent more serious cases of accidents is entirely possible from the view of hospital stakeholders. The pooling of aggregate data from multiple incident reporting systems can assist with formulating best practice recommendations. Incident reporting systems can also serve as a communication tool $[1,26,27]$. A comprehensive accident injuryprevention effort can be a targeted driving force from bottom up knowledge and safety value sharing and implementing in parallel with top down policy through a "leadership walk round" in a positive manner. Empowerment monitoring is needed to initiate and develop feedback loops to increase attention and resources dedicated to motivating practitioners to reduce accident injuries and focus on near miss reporting for prevention [28,29] and active surveillance of a safe-hospital culture, both to prevent accidents and near-misses in the hospital setting [30-32].

\section{Conclusion}

Accidents and near misses, unreported and underreported findings, showed trends of increasing rates of incident reports through hospital officers more active participation than previously, with an increased safety-culture organization resulting in reductions of severe accident case reports. Accidents and near-misses, underreported, still existed over 3 months, and were especially high regarding near-misses because hospital officers were not clear about concepts and how to integrate these into practice or faced transformative obstacles implementing real practices because of fear to report. Therefore, this study reflects the need for improvement in safety-organization culture end points and risk-marker identification and management with much more attention given to practices of experiential and knowledge-sharing among leaders and practitioners. Better prioritizing and implementation of reporting practices of nearmisses and incident reports of risk-markers as benchmarks for comparison within and between groups in tertiary hospitals is needed for improved reports with fewer accidents.

\section{Source of Financial Support}

The Fogarty International Center and the National Institute of Environmental Health Sciences of the National Institutes of Health, and the National Institute for Occupational Safety and Health of the U.S. Centers for Disease Control and Prevention; under Grant the Global Environmental and Occupational Health program awards. [1R24TW009560 and 4R24TW009558] Prof. Dr.Pornpimol Kongtip,PhD in Occupational Science.

\section{Acknowledgments}

This work was supported by the Fogarty International Center and the National Institute of Environmental Health Sciences of the National Institutes of Health, and the National Institute for Occupational Safety and Health of the U.S. Centers for Disease Control and Prevention. 


\section{References}

1 Vincent C (2015) The essentials of patient safety. (2nd Edtn), Wiley and Sons, West Sussex, United Kingdom.

2 Types of reportable incidents; RIDDOR-Reporting of Injuries, Diseases and Dangerous Occurrences Regulations.

3 Kjellén U (2000) Prevention of accidents through experience feedback. Taylor and Francis, London.

4 ACC (2015) How to implement safe workplace practices: A guide to workplace health and safety.

5 HSE (2015) Investigating accidents and incidents A workbook for employers, unions, safety representatives and safety professionals.

6 WHO (2009) Guidelines on hand hygiene in health care; First Global Patient Safety Challenge.

7 WHO (2016) Clean care is safer care.

8 WHO (2005) Draft guidelines for adverse event reporting and learning systems.

9 Kim KM, Kang SW, Kim YM, You M (2014) Nurses willingness to report near misses: A multilevel analysis of contributing factors. Soc Behav Pers 42: 1133-1146.

10 Conway H, Svenson J (1998) Occupational injury and illness rates. Monthly Lab Rev 121: 36-58.

11 Wolf ZR, Hughes R G (2008) Patient safety and quality: An evidencebased handbook for nurses "error reporting and disclosure".

12 Ariss SS (2003) Employee involvement to improve safety in the workplace: An ethical imperative. Am J Bus 18: 9-16.

13 Hughes RG (2008) Patient safety and quality: An evidence-based handbook for nurses.

14 The Joint Commission (2012) Improving patient and worker safety: Opportunities for synergy, collaboration and innovation.

15 Viwatwongkasem $C$, Satitvipawee $P$, Jareinpituk $S$, Soontornpipit $P$ (2013) Mixture models for estimating the number of drug users in thailand 2005-2007. Scientific Res 4: 1242-1250.

16 Viwatwongkasem C, Satitvipawee $P$, Jareinpituk S, Soontornpipit $P$ (2008) A comparison of population size estimators under the truncated count model with and without allowance for contaminations. Biom J 50: 1006-1021.

17 Clarke SP, Sloane DM, Aiken LH (2002) Effects of hospital staffing and organizational climate on needle stick injuries to nurses. Am J Public Health 92: 1115-1119.

18 Hanrahan NP, Kumar A, Aiken LH (2010) Adverse events associated with organizational factors of general hospital inpatient psychiatric care environments. Psychiatry Serv 6: 569-574.

19 US government accountability office (2014) Patient safety and quality improvement act of 2005-2014.

20 Crowley P (2014) Safety incident management policy the hse safety incident management policy.

21 White C (2016) Incident and serious incidents reporting and management policy, V10.

22 Emslie S (2016) Patient safety systems (PS) comprehensive accreditation manual for hospitals.

23 Kaplan HC, Brady PW, Dritz MC, Hooper DK, Linam WM, et al. (2010) The influence of context on quality improvement success in health care: a systematic review of the literature proposed on near misses. Milbank Q 88: 500-559.

24 Emslie S, Knox K, Pickstone M (2002) Improving patient safety:insights from american, australian and british healthcare.

25 Al-Ghemlas W (2004) Study on aramco berri gas plant safety management program. master of engineering report king fahd university of petroleum \& minerals.

26 Hopkins A (2010) Why BP ignored close calls at texas city.

27 Nemeth CP (2008) Improving healthcare team communication building on lessons from aviation and aerospace. Ashgate Publishing Limited, USA.

28 NERC Science of the environment (2015) Work related accident, incident, ill health and near miss reporting and investigation.

29 Valent F, Liva G, Bellomo F, de Corti D (2016) Ecological study on the association between characteristics of hospital units and the risk of occupational injuries and adverse events on the example of an italian teaching hospital. Int J Occup Med Environ Health 29: 149-159.

30 White D, Hays G, McSharry R, Giles J, Cheraghi-Sohi S, et al. (2015) Blame the Patient, Blame the doctor or blame the system? A metasynthesis of qualitative studies of patient safety in primary care.

31 Pham J C, Girard T, Pronovost P (2013) What to do with healthcare Incident Reporting Systems. J Public Health Research 2: 154-159.

32 Ekahau APAC (2015) Six ways to improve staff safety in healthcare. 